\title{
Development of a Tetracycline Resistant Strain of E. coli Sensitive to Ultraviolet Radiation
}

\author{
Meredith Joy Reesor ${ }^{\mathrm{a}}$, Isaac Joseph King ${ }^{\mathrm{a}}$, Jeffrey Michael Copeland ${ }^{\mathrm{a}}$
}

\begin{abstract}
Multidrug resistance bacteria pose a significant threat to human health and the efforts of the medical community. Given our reliance on antibiotics for therapeutic treatment of bacterial infections it is imperative to understand the mechanism by which bacteria develop antibiotic resistance. In the present report we develop a strain of Escherichia coli capable of resisting high levels of tetracycline and other protein synthesis inhibitors. Furthermore the tetracycline resistant strain is approximately $1 / 3^{\text {rd }}$ in length and is sensitive to UV radiation.
\end{abstract}

Keywords: Antibiotic resistance, UV radiation, Tetracycline

\section{Introduction}

Tetracycline is a broad spectrum antibiotic that acts against both gram-positive and gram- negative bacteria and was derived from the soil bacterium Streptomyces aureofaciens (Chopra \& Roberts, 2001). Since its initial isolation, over twelve tetracycline derivatives have been discovered, and there are currently more undergoing clinical trials. In the past, tetracycline was a very effective outpatient drug because it is relatively inexpensive, has low toxicity, can be taken orally and has few side effects. However, within the past few decades, tetracycline and its derivatives are used less frequently in outpatient treatment due to the appearance of resistance in medically important bacterial strains, such as Streptococcus pneumoniae, Neisseria gonorrhoeae, Escherichia coli and Klebsiella pneumoniae (Alekshun \& Levy, 2007; Montanari, Cochetti, Mingoia, \& Varaldo, 2003; Morse, Johnson, Biddle, \& Roberts, 1986). Although the usefulness of tetracycline against infection and disease has been diminished, it is still administered commonly for treating acne and rosacea. Less commonly, tetracycline drugs are effective in treating malaria, bubonic plague, Rickettsia, infections caused by chlamydia, as well as many other respiratory, skin, urinary and gastrointestinal diseases (Speer, Shoemaker, \& Salyers, 1992). As is the case with many other antibiotics, the loss of effectiveness of tetracycline is troublesome as it diminishes the drug arsenal available for treating bacterial infections.

It has been well noted that tetracycline resistance can be found pervasively, in clinical and natural populations, as well in selected and non-selected isolates of E. coli. The importance of this wide spread resistance is not reflective of its banality, but rather the overabundance of tetracycline usage, especially on farm animals, and the relative ease of transferring resistance genes. It has been estimated that during the 1990s, 3.5 million $\mathrm{kg}$ of tetracycline was used per year in the US, just for animal growth practices and for prophylactic treatment in livestock (Chopra \& Roberts, 2001). It is of no wonder that tetracycline resistance is prevalent in bacterial isolates taken from livestock and humans (Ahmed, Clegg, Williams, Baptiste, \& Bennett, 2010; Johnson \& Adams, 1992; Karami, Nowrouzian, Adlerberth, \& Wold, 2006; Villedieu et al., 2003). Additional reasons for the pervasiveness of tetracycline resistance could be due to the fact that the resistance genes, for example the most common tet(A) and tet(B), are often found in conjunction with other resistance factors on mobile transposons or plasmids. Resistance to tetracycline is often found in association with streptomycin and ampicillin, and selection for resistance to one antibiotic could potentially lead to resistance to more (Vignaroli et al., 2012; Ziebell et al., 2011). Finally, persistence of tetracycline resistant bacteria has been demonstrated despite the removal of tetracycline from the system (Smith, 1975). One potential means of explaining this persistence is the potential for bacteria to reduce or even eliminate any fitness cost associated with drug resistance and thus placing no selective pressure for the removal of resistant bacteria in a population. Tetracycline resistant $E$. coli grown in the presence of tetracycline for approximately 500 generations co-evolved mechanisms that rendered fitness advantages compared to tetracycline sensitive strains, even in the absence of the drug (Bouma \& Lenski, 1988; Lenski, 2010). Despite the commonality of tetracycline resistance in a multitude of different animal and human populations, this frequency does not detract from the importance of understanding of tetracycline resistance but rather underscore the necessity for further exploration.

There are currently three known mechanisms by which bacteria develop resistance to tetracycline: efflux proteins, ribosomal protection proteins and enzymatic inactivation. Efflux proteins are the most extensively studied of these resistance mechanisms. They function to export tetracycline from the cell, thus reducing the concentration of tetracycline in the cell and protecting the targeted bacteria (Roberts, 2005). Efflux proteins are endogenously found in a wide spectrum of bacteria, and confer resistance to tetracycline and not glycylcyclines or minocycline (Chopra \& Roberts, 2001). Efflux proteins use a variety of methods for the removal of substances, including antiport mechanisms or the hydrolysis of ATP (Piddock, 2006). These naturally occurring efflux proteins may also play a role in bacterial pathogenicity by aiding in colonization and excretion of virulence factors (Piddock, 2006). There are five families of efflux pump proteins associated with multidrug resistance; the major facilitator superfamily (MFS), the ATP binding cassette superfamily $(\mathrm{ABC})$, the small multidrug resistance family (SMR), the multidrug and toxic-compound extrusion family (MATE) and the resistance nodulation division family (RND). 
A single organism or cell can express efflux protein pumps from several families or different efflux protein pumps from within a family. Escherichia coli expresses the AcrA, AcrB and TolC proteins, which together form a RND efflux pump (Alekshun \& Levy, 2007).

Given the reliance on antibiotics in our medical system and usage in livestocks, it is worth examining the mechanisms in which resistance to tetracycline and its derivatives develops. In this article, we develop a tetracycline resistant bacterial strain, which shows resistance to ribosomal blocking drugs and is sensitive to UV radiation.

\section{Results}

The first goal of our project was to develop a K12 strain of E. coli capable of resisting high levels of tetracycline. To achieve this, E. coli was sequentially cultured on increasing amounts of the antibiotic. Eventually the strain was capable of growth in the presence of $200 \mu \mathrm{g} / \mathrm{ml}$ tetracycline. To confirm the tetracycline resistance and to test for the ability to resist other antibiotics capable of inhibiting protein synthesis, an antibiotic disc test was carried out. The starting strain of $E$. coli, which was tetracycline sensitive, was plated first with a tetracycline antibiotic disc. This resulted in a circle of destroyed bacteria around the disc with a radius of two centimeters. Afterwards, tetracycline resistant E. coli was plated with a tetracycline antibiotic disc and no ring was observed; this points to effective resistance. Similarly, tetracycline sensitive and tetracycline resistant $E$. coli were tested against a series of drugs that inactive the ribosome. Neomycin, streptomycin and gentamicin are all aminoglycosides and erythromycin is a macrolide. The tetracycline resistant $E$. coli displayed multidrug resistance to all of these protein synthesis inhibitors tested (Figure 1).

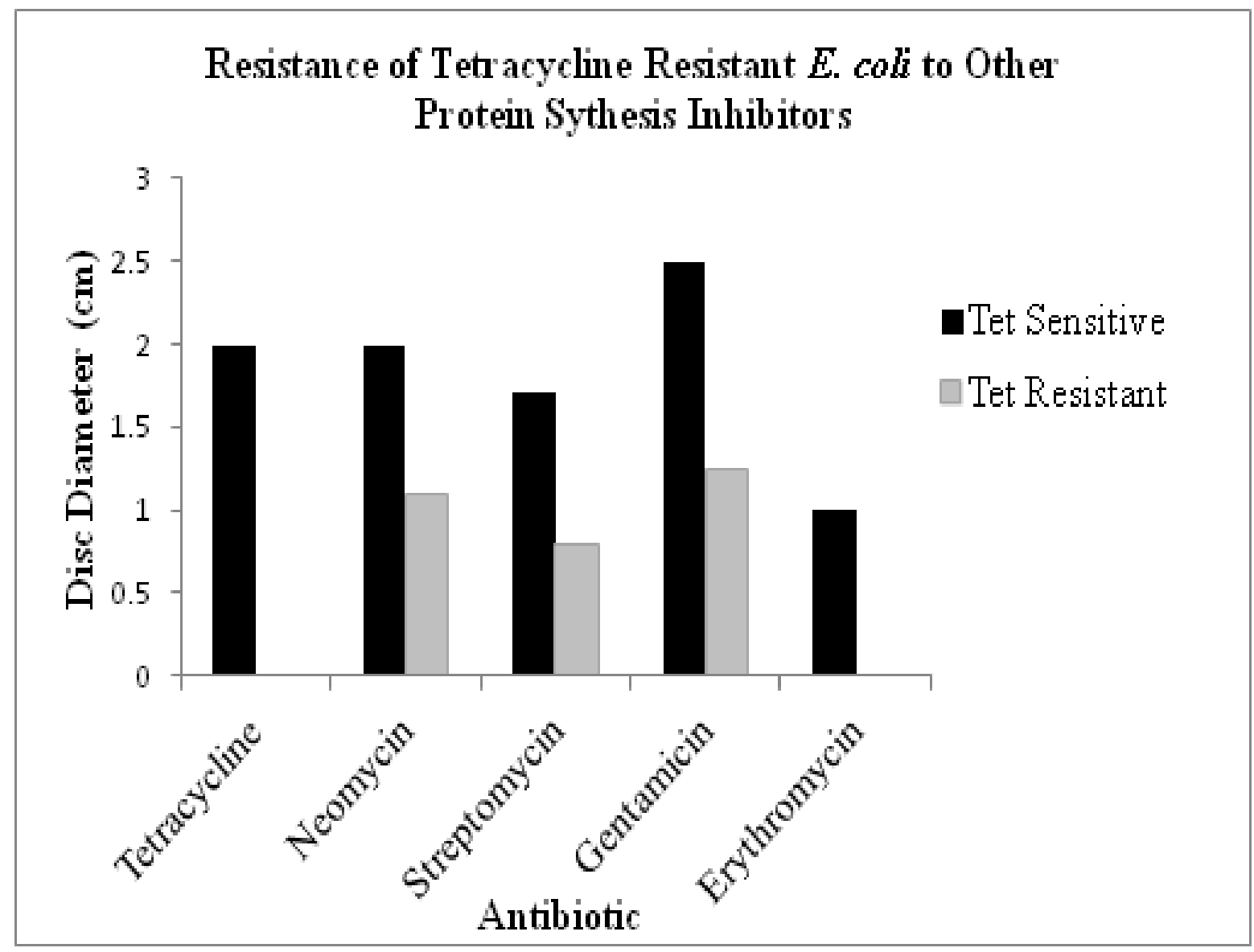

Figure 1. Multidrug resistance displayed by the tetracycline resistant $E$. coli. Resistance is measured in centimeters from the antibiotic disc. 
To compare the morphological appearance of tetracycline sensitive and tetracycline resistant $E$. coli, both strains were grown on 5\% sheep blood agar and examined by eye. The tetracycline resistant strain appeared glossier and smoother in comparison to the tetracycline sensitive strain when examined (Figure 2). Gram stains were performed on tetracycline resistant and sensitive E. coli to determine any morphological differences. As seen with a microscope, the tetracycline resistant $E$. coli appear on average $1 / 3^{\text {rd }}$ in length in comparison with the tetracycline sensitive E. coli (Figure $3)$.

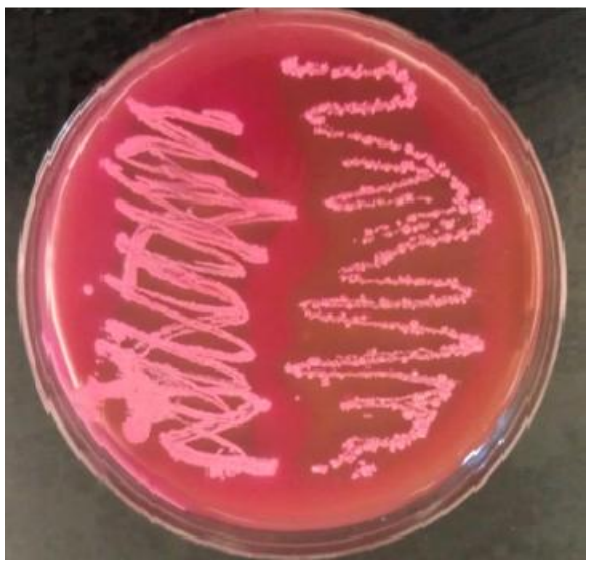

Figure 2. Tetracycline resistant $E$. coli cultures are glossy when streaked on 5\% sheep blood agar (TSA agar). Tetracycline resistant $E$. coli is on left and tetracycline sensitive is on the right side of the plate.

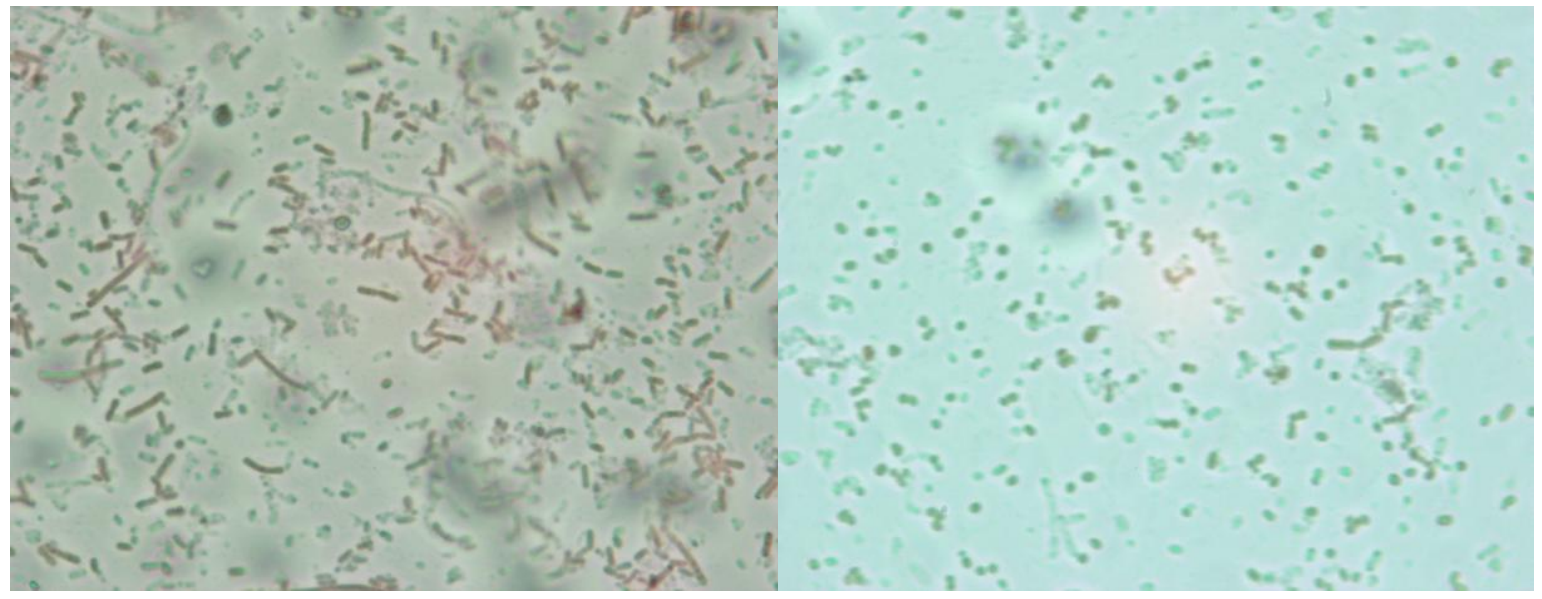

Figure 3.Tetracycline resistant $E$. coli are approximately $1 / 3^{\text {rd }}$ length of tetracycline sensitive E. coli. The tetracycline sensitive strain is the left panel while the tetracycline resistant is the right one.

Initially we were eager to test the genetic components conferring tetracycline resistance through UV-induced mutagenesis of the genome and restoration of tetracycline sensitivity. The results however show that the combination of UV radiation and tetracycline kills the resistant strain of bacteria quite effectively (Figure 4). Given that in many situations the mere presence of antibiotics is sufficient to introduce DNA mutations, our results suggests that the combination of UV radiation and tetracycline cooperatively compromise the integrity of the genome and bacterial growth (Dwyer, Kohanski, \& Collins, 2009). 


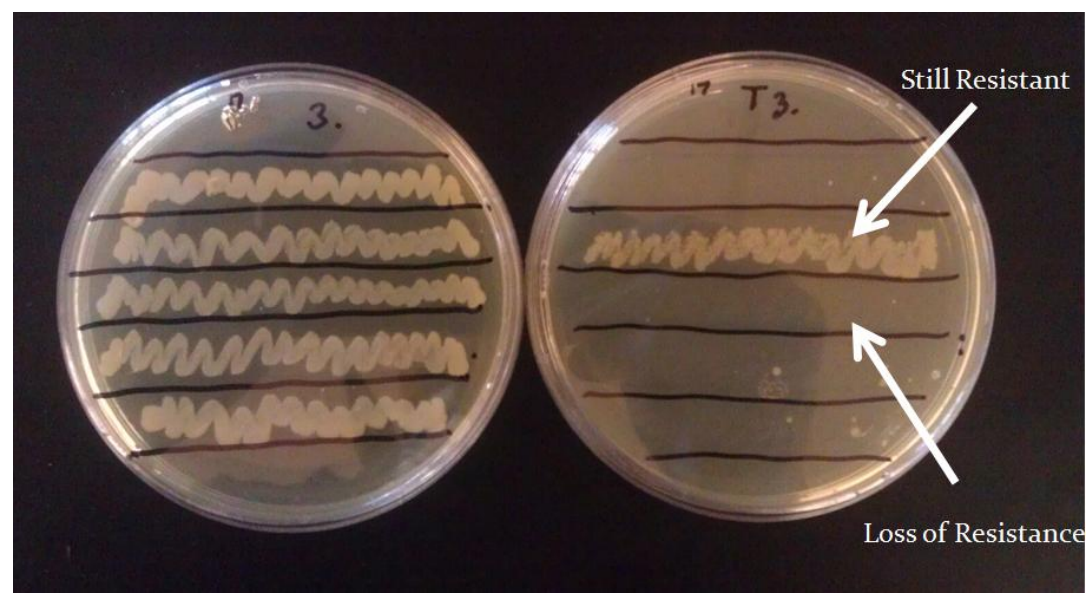

Figure 4. Tetracycline resistant $E$. coli is sensitive to UV-irradiation as compared to the controls. UV irradiated strains are grown in the absence of tetracycline (left plate) or in the presence of tetracycline (right plate).

\section{Discussion}

One of the major problems facing modern medicine is the rapid increase in antibiotic resistant bacteria. To understand the cellular mechanism underlying resistance, we grew a K12 strain of $E$. coli on increasing amounts of tetracycline. Many bacteria have developed resistance to tetracycline, including E. coli, Streptococcus pneumoniae, Salmonella enterica, Staphylococcus aureus, and members of the Shigella genus (Chopra \& Roberts, 2001). Though tetracycline is administered to achieve serum levels of 5 $\square \mathrm{g} / \mathrm{ml}$, we grew our $E$. coli to resist $200 \mu \mathrm{g} / \mathrm{ml}$ since some clinical isolates of other bacteria, notably Neisseria gonorrhoeae have the ability to resist up to $64 \mu \mathrm{g} / \mathrm{ml}$ (Chopra \& Roberts, 2001; Morse et al., 1986).

To verify that our E. coli was indeed tetracycline resistant and to determine resistance to other antibiotics, we tested our strain using a standard Kirby-Bauer disc assay. In this assay, the E. coli appeared to be completely resistant to the tetracycline and additionally to erythromycin. Some degree of additional resistance was seen against neomycin, streptomycin and gentamicin. All of the tested antibiotics are known to prohibit ribosomal function, but with varied modes of action. It has been shown that tetracycline binds to the $16 \mathrm{~S}$ RNA subunit in the $30 \mathrm{~S}$ subunit, near the aminoacyl-tRNA binding site (Brodersen et al., 2000; Pioletti et al., 2001). Aminoglycosides have been isolated from Streptomyces genus, like the antibiotic streptomycin, and have been shown to bind to the 16S RNA subunit, interfering with aminoacyltRNA selection and proof-reading (Carter et al., 2000). On the other hand, erythromycin, a macrolide, targets the $23 \mathrm{~S}$ rRNA found in the large 50S subunit (Schlünzen et al., 2001). Though the antibiotics tested all counteract the activity of the ribosome by various methods, the generation of a broad acting tetracycline resistant $E$. coli might provide some insight into the nature of the resistance.

As previously discussed, bacteria employ different means to protect themselves against tetracycline, with the most common being efflux proteins. Although there is extensive research on the mechanisms specifically for tetracycline resistance, evidence is only now growing about how bacteria can become multidrug resistant to the combination of tetracycline and aminoglycosides. Previous studies have shown methicillin resistant Staphyloccocus aureus and erythromycin resistant Streptococcus pneumoniae can develop resistance to tetracycline by either acquiring the ability for active efflux or ribosomal protection (Montanari et al., 2003; Trzcinski, Cooper, Hryniewicz, \& Dowson, 2000). It is interesting to note that in spore forming bacteria isolated from soil cultures, few if any, were resistant to the combination of gentamicin, tetracycline, erythromycin and neomycin (D'Costa, McGrann, Hughes, \& Wright, 2006).

Although we never directly tested the activity nor the levels of any of the efflux proteins we suspect that alterations of endogenous efflux genes accounts for the increased activity of tetracycline resistance. First of all, efflux systems have been important in conferring multidrug resistance and the export of a range of antibiotics. Removal of TolC, the outer membrane component of the RND efflux system, sensitizes $E$. coli to both tetracycline and erythromycin (Sulavik et al., 2001). Activity of AcrAB, the inner membrane component of the RND efflux system, was necessary and sufficient for resistance to tetracycline, $\beta$-lactams and chloroamphenicol (Nishino \& Yamaguchi, 2001; Okusu, Ma, \& Nikaido, 1996). Removal of the negative regulators of the RND system, specifically for the AcrAB gene operon, can affect gene expression and multidrug resistance (Nikaido, 2009). Secondly, lateral gene transfer, a known means of spreading antibiotic resistance elements, seems unlikely since our system was developed in a laboratory setting without any tetracycline resistant bacteria, and our $E$. coli did not come in contact with any other microbe. Ribosomal protection proteins offer the other possibility for the developed tetracycline resistance seen, but we suspect that they do not impart any protection against the antibiotics tested. Tet(O) and $\operatorname{Tet}(\mathrm{M})$ are the most characterized ribosomal protection proteins. The $\operatorname{tet}(O)$ and $\operatorname{tet}(M)$ most likely arose from 
Streptomyces rimosus and are spread through lateral gene transfer (Connell, Tracz, Nierhaus, \& Taylor, 2003). Given that our tetracycline resistant bacteria arose de novo, it is unlikely that lateral gene transfer is the means by which resistance developed. Furthermore, Tet $(\mathrm{O})$ functions to remove tetracycline from the A-site on the ribosome, which is not a targeted site of erythromycin (Spahn et al., 2001). For the reasons stated above, we suspect resistance to tetracycline in our E. coli developed from increased activity or increased gene expression of efflux pumps.

The sensitization of our tetracycline resistant E. coli to UV irradiation is an interesting result and warrants comments. In addition to the model in which resistance develops from pre-existing genetic variants within a population, recent studies have shown that low levels of DNA damage can facilitate genetic evolution and antibiotic resistance through the activation of the SOS DNA repair system (Dwyer et al., 2009; Kohanski, DePristo, \& Collins, 2010). The generation of reactive oxygen species (ROS), has been shown to be a direct consequence of the administration of several antibiotics, which is particularly interesting given the fact that the detection of ROS by the SoxR regulator leads to the eventual transcription of the tolC and $a c r A B$ genes (Kohanski et al., 2010; Pomposiello \& Demple, 2001). Thus, while it seems that the DNA damage initiated through the presence of antibiotics is beneficial to the development of resistance, our results suggest that the combination of antibiotics with UV irradiation actually sensitizes the cells. We would like to speculate here that this sensitization is a result of the overactivity of PolV, a known error prone polymerase initiated by both ROS and UV irradiation (Tang et al., 2000). We suspect that the combination of tetracycline administration and UV radiation activates PolV, induces an error prone repair system, and incorporates many nucleotide changes into the bacterial genome. Thus, PolV while trying to maintain genomic integrity actually reduces cell viability.

In conclusion, we have developed an E. coli strain capable of resisting multiple antibiotics by selecting cells tolerant of increasing amounts of tetracycline. Our strain of bacteria also displayed sensitivity to UV radiation and appears shorter under a compound microscope. While the current work presented in this article reveals a multidrug resistant $E$. coli strain, future experiments will explore the molecular mechanism behind this resistance, which might include efflux or ribosomal protection. We hope that an improved understanding of the development of antibiotic resistance will eventually lead to new therapies for bacterial infections.

\section{Experimental Procedures}

\section{Growth and Characterization}

Tetracycline resistant Escherichia coli was developed by streaking a K12 strain every $2-3$ days on to LB agar plates containing increasing amounts of tetracycline. Concentrations of tetracycline plates used were as follows: $0.1,0.5,1,2,10,50,100,200 \square \mathrm{g} / \mathrm{mL}$. Since many plasmids confer resistance around $100 \square \mathrm{g} / \mathrm{mL}$, our tetracycline resistant $E$. coli was eventually able to grow in the presence of $200 \square \mathrm{g} / \mathrm{mL}$ of tetracycline. To test for susceptibility to other antibiotics, the tetracycline resistant $E$. coli was used in a standard Kirby-Bauer disc assay. Overnight growth of E. coli was plated on standard LB agar plates and then incubated in the presence of disks containing the following antibiotics: tetracycline $(30 \mu \mathrm{g})$, neomycin $(30 \mu \mathrm{g})$, streptomycin $(10 \mu \mathrm{g})$, gentamicin $(10 \mu \mathrm{g})$ and erythromycin $(15 \mu \mathrm{g})$. Drug resistance was quantified by measuring the zone of growth inhibition surrounding each antibiotic disk after 48 hours of incubation (Chess, 2009). A standard Gram stain was performed on both tetracycline sensitive and resistant $E$. coli, and then photographed by a SPOT Imaging Solutions (Sterling Heights, MI) camera over a Nikon E200- LED (Melville, NY) microscope. Growth on 5\% sheep blood was used to examine colony appearance and luster.

\section{Radiation}

The tetracycline resistant strain of $E$. coli was grown to $\mathrm{OD}_{600}=0.4$ and then plated on a standard LB agar plate. The plated bacteria were then UV-irradiated by holding the plates $22 \mathrm{~cm}$ above an UV light box $(312 \mathrm{~nm})$ for 30 seconds and then incubating for 24 hours. Twenty-five random colonies from the incubated plates were streaked again onto tetracycline LB agar plates and non-tetracycline LB agar plates.

\section{References}

Ahmed, M. O., Clegg, P. D., Williams, N. J., Baptiste, K. E., \& Bennett, M. (2010). Antimicrobial resistance in equine faecal Escherichia coli isolates from North West England. Annals of clinical microbiology and antimicrobials, 9(1), 12.

Alekshun, M. N., \& Levy, S. B. (2007). Molecular mechanisms of antibacterial multidrug resistance. Cell, 128(6), 1037-1050.

Bouma, J. E., \& Lenski, R. E. (1988). Evolution of a bacteria/plasmid association.

Brodersen, D. E., Clemons Jr, W. M., Carter, A. P., MorganWarren, R. J., Wimberly, B. T., \& Ramakrishnan, V. (2000). The structural basis for the action of the antibiotics tetracycline, pactamycin, and hygromycin B on the $30 \mathrm{~S}$ ribosomal subunit. Cell, 103(7), 1143-1154.

Carter, A. P., Clemons, W. M., Brodersen, D. E., MorganWarren, R. J., Wimberly, B. T., \& Ramakrishnan, V. (2000). Functional insights from the structure of the $30 \mathrm{~S}$ ribosomal subunit and its interactions with antibiotics. Nature, 407(6802), 340-348.

Chopra, I., \& Roberts, M. (2001). Tetracycline antibiotics: mode of action, applications, molecular biology, and epidemiology of bacterial resistance. Microbiology and Molecular Biology Reviews, 65(2), 232-260.

Connell, S. R., Tracz, D. M., Nierhaus, K. H., \& Taylor, D. E. (2003). Ribosomal protection proteins and their mechanism of tetracycline resistance. Antimicrobial agents and chemotherapy, 47(12), 3675-3681.

D'Costa, V. M., McGrann, K. M., Hughes, D. W., \& Wright, G. D. (2006). Sampling the antibiotic resistome. Science, 311(5759), 374-377. 
Dwyer, D. J., Kohanski, M. A., \& Collins, J. J. (2009). Role of reactive oxygen species in antibiotic action and resistance. Current opinion in microbiology, 12(5), 482-489.

Johnson, R., \& Adams, J. (1992). The ecology and evolution of tetracycline resistance. Trends in Ecology \& Evolution, 7(9), 295-299.

Karami, N., Nowrouzian, F., Adlerberth, I., \& Wold, A. E. (2006). Tetracycline resistance in Escherichia coli and persistence in the infantile colonic microbiota. Antimicrobial agents and chemotherapy, 50(1), 156-161.

Kohanski, M. A., DePristo, M. A., \& Collins, J. J. (2010). Sublethal antibiotic treatment leads to multidrug resistance via radical-induced mutagenesis. Molecular cell, 37(3), 311-320.

Lenski, R. E. (2010). Bacterial evolution and the cost of antibiotic resistance. International Microbiology, 1(4), 265-270.

Montanari, M. P., Cochetti, I., Mingoia, M., \& Varaldo, P. E. (2003). Phenotypic and molecular characterization of tetracycline-and erythromycin-resistant strains of Streptococcus pneumoniae. Antimicrobial agents and chemotherapy, 47(7), 2236-2241.

Morse, S. A., Johnson, S., Biddle, J., \& Roberts, M. (1986). High-level tetracycline resistance in Neisseria gonorrhoeae is result of acquisition of streptococcal tetM determinant. Antimicrobial agents and chemotherapy, 30(5), 664-670.

Nikaido, H. (2009). Multidrug resistance in bacteria. Annual review of biochemistry, 78, 119.

Nishino, K., \& Yamaguchi, A. (2001). Analysis of a complete library of putative drug transporter genes in Escherichia coli. Journal of Bacteriology, 183(20), 5803-5812.

Okusu, H., Ma, D., \& Nikaido, H. (1996). AcrAB efflux pump plays a major role in the antibiotic resistance phenotype of Escherichia coli multiple-antibioticresistance (Mar) mutants. Journal of Bacteriology, 178(1), 306-308.

Piddock, L. J. (2006). Multidrug-resistance efflux pumps? not just for resistance. Nature Reviews Microbiology, 4(8), 629-636.

Pioletti, M., Schlünzen, F., Harms, J., Zarivach, R., Glühmann, M., Avila, H., . . . Jacobi, C. (2001). Crystal structures of complexes of the small ribosomal subunit with tetracycline, edeine and IF3. The EMBO Journal, 20(8), 1829-1839.

Pomposiello, P. J., \& Demple, B. (2001). Redox-operated genetic switches: the SoxR and OxyR transcription factors. Trends in biotechnology, 19(3), 109-113.

Roberts, M. C. (2005). Update on acquired tetracycline resistance genes. FEMS microbiology letters, 245(2), 195-203.
Schlünzen, F., Zarivach, R., Harms, J., Bashan, A., Tocilj, A., Albrecht, R., . . . Franceschi, F. (2001). Structural basis for the interaction of antibiotics with the peptidyl transferase centre in eubacteria. Nature, 413(6858), 814-821.

Smith, H. W. (1975). Persistence of tetracycline resistance in pig E. coli. Nature, 258(5536), 628-630.

Spahn, C. M., Blaha, G., Agrawal, R. K., Penczek, P., Grassucci, R. A., Trieber, C. A., . . . Frank, J. (2001). Localization of the ribosomal protection protein Tet $(\mathrm{O})$ on the ribosome and the mechanism of tetracycline resistance. Molecular cell, 7(5), 1037-1045.

Speer, B. S., Shoemaker, N. B., \& Salyers, A. A. (1992). Bacterial resistance to tetracycline: mechanisms, transfer, and clinical significance. Clinical microbiology reviews, 5(4), 387-399.

Sulavik, M. C., Houseweart, C., Cramer, C., Jiwani, N., Murgolo, N., Greene, J., . . . Hare, R. (2001). Antibiotic Susceptibility Profiles ofEscherichia coli Strains Lacking Multidrug Efflux Pump Genes. Antimicrobial agents and chemotherapy, 45(4), 1126-1136.

Tang, M., Pham, P., Shen, X., Taylor, J.-S., O'Donnell, M., Woodgate, R., \& Goodman, M. F. (2000). Roles of E. coli DNA polymerases IV and $\mathrm{V}$ in lesiontargeted and untargeted SOS mutagenesis. Nature, 404(6781), 1014-1018.

Trzcinski, K., Cooper, B. S., Hryniewicz, W., \& Dowson, C. G. (2000). Expression of resistance to tetracyclines in strains of methicillin-resistant Staphylococcus aureus. Journal of Antimicrobial Chemotherapy, 45(6), 763-770.

Vignaroli, C., Luna, G., Rinaldi, C., Di Cesare, A., Danovaro, R., \& Biavasco, F. (2012). New sequence types and multidrug resistance among pathogenic Escherichia coli isolates from coastal marine sediments. Applied and environmental microbiology, 78(11), 39163922.

Villedieu, A., Diaz-Torres, M., Hunt, N., McNab, R., Spratt, D., Wilson, M., \& Mullany, P. (2003). Prevalence of tetracycline resistance genes in oral bacteria. Antimicrobial agents and chemotherapy, 47(3), 878-882.

Ziebell, K., Johnson, R., Kropinski, A., Reid-Smith, R., Ahmed, R., Gannon, V., . . . Boerlin, P. (2011). Gene cluster conferring streptomycin, sulfonamide, and tetracycline resistance in Escherichia coli O157: H7 phage types 23, 45, and 67. Applied and environmental microbiology, 77(5), 1900-1903. 